\title{
Dynamic Quantitative Contrast-enhanced Ultrasound for Assessment of Cilostazol Induced Pro-angiogenic Therapeutic Effects on Skeletal Muscle Microcirculation in Peripheral Arterial Disease
}

\author{
Kauczor HU1, Amarteifio $E^{1,3}$, Partovi $S^{2}$, Weber MA ${ }^{1,3}$, Macys $L^{1,3}$, Delorme $S^{3}$, Krix $\mathbf{M}^{3,4}$, Böckler $D^{5}$ and Demirel $S^{5 *}$ \\ ${ }^{1}$ University Hospital of Heidelberg, Department of Diagnostic and Interventional Radiology, Heidelberg, Germany \\ ${ }^{2}$ Department of Radiology, University Hospitals Case Medical Center, Case Western Reserve University, Cleveland, Ohio, USA \\ ${ }^{3}$ Department of Radiology, German Cancer Research Center, Heidelberg, Germany \\ ${ }^{4}$ Bracco Imaging Germany, Konstanz, Germany \\ ${ }^{5}$ University Hospital of Heidelberg, Department of Vascular and Endovascular Surgery, Heidelberg, Germany
}

\begin{abstract}
Purpose: To assess the effect of cilostazol on muscular microperfusion in patients with peripheral artery occlusive disease (PAOD) using dynamic quantitative contrast-enhanced ultrasound (CEUS).

Materials and Methods: 20 patients with PAOD, Rutherford classification grade I, category III were evaluated performing CEUS with the arterial occlusion paradigm, ankle-brachial-index (ABI) and treadmill walking distance test before and after oral treatment with cilostazol for three months. Microperfusion of the calf skeletal muscle was assessed using quantitative CEUS parameters which were extracted from time signal-intensity curves (TIC).

Results: 10 patients had to be taken off medication secondary to side effects. Maximum CEUS signal enhancement prior to therapy was $104 \pm 69 \sim \mathrm{mL}$ compared to $114 \pm 68 \sim \mathrm{mL}$ post treatment $(P=0.26)$. tmax was 34 $\pm 20 \mathrm{~s}$ before and $36 \pm 23 \mathrm{~s}$ post treatment $(\mathrm{P}=0.51)$. Maximum slope of the TIC $(\mathrm{m} 2)$ prior to therapy was $6.06 \pm$ $5.55 \sim \mathrm{mL} / \mathrm{s}$ and revealed to be $5.12 \pm 3.30 \sim \mathrm{mL} / \mathrm{s}$ post treatment $(P=0.62)$. Area under the curve after the occlusion (AUCpost) was $3975.38 \pm 2856.52 \sim \mathrm{mL} / \mathrm{s}$ prior to cilostazol therapy compared to $4210.98 \pm 2816.33 \sim \mathrm{mL} / \mathrm{s}$ after therapy $(P=0.39)$. The values for $A B I$ and treadmill walking distance test did not differ significantly differ, either.

Conclusion: In patients with PAOD, medical therapy with cilostazol seems not to be associated with significant improvement of muscular microperfusion as assessed by dynamic quantitative CEUS. This study demonstrates the feasibility of the CEUS technique to evaluate response to pro-angiogenic drug treatment and confirmation of results in a larger population is warranted.
\end{abstract}

Keywords: Muscle perfusion; Contrast-enhanced ultrasound; CEUS; Transient arterial occlusion; Peripheral arterial occlusive disease; PAOD; Cilostazol

\section{Compliance with Ethical Standards}

This prospective study was IRB approved and is HIPAA compliant as well as in accordance with the declaration of Helsinki. Written informed consent was obtained from all subjects prior to enrollment. This study did not receive funding.

\section{Introduction}

The most commonly used diagnostic tools for the management of patients with peripheral arterial occlusive disease (PAOD) are clinical examination, ankle-brachial index (ABI), walking distance, color coded and pulsed wave duplex ultrasound, magnetic resonance angiography (MRA), computed tomography angiography (CTA), and conventional angiography for therapeutic purposes [1-3]. These radiological techniques have in common that they primarily focus on the macrovasculature evaluation including stenotic and occluded sites. However they are unable to assess the microvasculature which plays a fundamental role in the PAOD pathogenesis since the skeletal muscle and the skin are the functional end-organs causing eventually the clinical picture in this patient population [1]. Therefore microperfusion assessment is key in PAOD patients to assess the effects of proangiogenic drugs on the functional end organ of interest, namely the skeletal muscle $[2,3]$.

Low-mechanical index quantitative contrast-enhanced ultrasound
(low-MI CEUS) in combination with transient arterial occlusion as an evolving paradigm has been introduced as a useful und suitable method to evaluate changes in the micro-perfusion of skeletal muscles [1-6]. Further it should be considered is a cost effective and therefore clinically attractive imaging method [1].

Treatment of symptomatic PAOD patients includes supervised exercise programmes, endovascular and surgical revascularization as well as pharmaceutical treatment such as cilostazol, which leads to phosphodiesterase-III-mediated increase of intracellular adenosine monophosphate resulting in vasodilation [1-3]. Treatment response is usually evaluated with clinical examination (improvement of symptoms, pulse palpation) combined with a treadmill walking distance test, $\mathrm{ABI}$ or duplex ultrasound $[1,2]$. Particularly the clinical

*Corresponding author: Demirel S, Department of Vascular and Endovascular Surgery, University of Heidelberg, Im Neuenheimer Feld 110, 69120 Heidelberg, Germany, Tel: 0049-6221-566249; Fax: 0049-6221-565423; E-mail: serdar.demirel@med.uni-heidelberg.de

Received August 02, 2016; Accepted August 12, 2016; Published August 20 2016

Citation: Kauczor HU, Amarteifio E, Partovi S, Weber MA, Macys L, et al. (2016) Dynamic Quantitative Contrast-enhanced Ultrasound for Assessment of Cilostazol Induced Pro-angiogenic Therapeutic Effects on Skeletal Muscle Microcirculation in Peripheral Arterial Disease. J Vasc Med Surg 4: 284. doi: 10.4172/23296925.1000284

Copyright: @ 2016 Kauczor HU, et al. This is an open-access article distributed under the terms of the Creative Commons Attribution License, which permits unrestricted use, distribution, and reproduction in any medium, provided the original author and source are credited. 
Citation: Kauczor HU, Amarteifio E, Partovi S, Weber MA, Macys L, et al. (2016) Dynamic Quantitative Contrast-enhanced Ultrasound for Assessment of Cilostazol Induced Pro-angiogenic Therapeutic Effects on Skeletal Muscle Microcirculation in Peripheral Arterial Disease. J Vasc Med Surg 4: 284. doi: 10.4172/2329-6925.1000284

Page 2 of 6

examination portion is harboring a subjective component and can be prone to biases, limiting reliability and reproducibility of the treatment response evaluation [7-15].

Purpose of the study was to investigate quantitatively the treatment response of pharmaceutical therapy with cilostazol in patients with PAOD on a microperfusion level by using dynamic low-MI CEUS combined with the paradigm of transient arterial occlusion and to compare CEUS parameters evaluated at early follow up with standard clinical tests and outcome. This was a longitudinal prospective study.

\section{Material and Methods}

\section{Study population}

Between October 2012 and October 2014, 20 patients (mean age 63 years, range 53-73 years; mean weight $80.2 \mathrm{~kg}$, range $54.0-134.0$ $\mathrm{kg}$ ) with PAOD, Rutherford classification grade I, category III were prospectively enrolled in a longitudinal clinical study.

The study was approved by the local ethical committee and review board and was performed according to the declaration of Helsinki [1]. The study was HIPAA compliant. Written informed consent was obtained from all subjects prior to enrollment.

Secondary to safety precautions patients with side effects from the cilostazol therapy in the first 14 days were immediately taken off the medication and not included in the study evaluation. From the 20 patients enrolled, 10 patients had to be excluded because of early (within the first 14 days) side effects of the therapy (Table 1) and cilostazol were stopped immediately. From the 20 initially enrolled patients, 13 patients were under single acetylsalicylic acid therapy (100 mg/day), 4 patients were on dual antiplatelet therapy with acetylsalicylic acid and clopidogrel 75 mg/day (Plavix ${ }^{\circledR}$, Sanofi-aventis, Paris, France) and 1 patient was treated with acetylsalicylic acid and rivaroxaban $20 \mathrm{mg} /$ day (Xarelto ${ }^{\circledR}$, Bayer AG, Leverkusen, Germany); 2 further patients were treated with clopidrogel only $75 \mathrm{mg} /$ day. The patients with double anti platelet medication stopped one anti platelet medication at least three months before beginning of medication with cilostazol. Other medication that would influence the P-450-enzymes CYP3A4 and CYP2C19 (particularly simvastatin) was replaced in order to minimize confounding factors in this prospective study. Exclusion criteria were severe heart failure, acute myocardial infarction, severe pulmonary hypertension, chronic obstructive pulmonary disease, moderate to severe renal or moderate to severe hepatic insufficiency. ABI and standardized treadmill test $(3.5 \mathrm{~km} / \mathrm{h} ; 12.5 \%$ ascending slope; $\mathrm{S} 2$ the maximum walking distance in meters; S1 - free of pain walking distance in meters) were evaluated before and 3 months after initiation of therapy with cilostazol (Pletal ${ }^{\mathbb{R}}$, Otsuka Pharmaceutical Co. Tokyo, Japan) [16-20].

\begin{tabular}{|c|c|}
\hline Patient number & Side effects \\
\hline$\# 2$ & Atrial fibrillation \\
\hline$\# 5$ & Forgetfulness \\
\hline$\# 7$ & Epistaxis \\
\hline$\# 10$ & Ecchymosis \\
\hline$\# 13$ & Pressure in chest region; dizziness \\
\hline$\# 14$ & Tachycardia; dyspnoea \\
\hline$\# 16$ & Dizziness \\
\hline$\# 17$ & Myocardial infarction \\
\hline$\# 20$ & Dizziness \\
\hline
\end{tabular}

Table 1: Observed early side effects ( $<14$ days) during cilostazol therapy.
All 20 patients had at least one haemodynamically relevant stenosis ( $>70 \%$ diameter reduction) of the femoropopliteal arterial segment. The patients had impaired quality of life due to intermittent leg pain that exacerbated with increasing walking distance and the patients were unable to complete individual walking training schedules. Since additional conservative treatment, such as cessation of smoking and reduction of cardiovascular risk factors, had failed to improve the individual symptoms, pharmaceutical therapy with cilostazol was considered as next treatment in line according to the guidelines of the American College of Cardiology Foundation and the American Heart Association [1]. The dose of cilostazol was $100 \mathrm{mg}$ b.i.d.

\section{Contrast-enhanced ultrasound}

Ultrasonography was performed by an experienced board certified radiologist with sub-specialization in musculoskeletal radiology and ultrasound (E.A.). The ultrasound examination was performed using a Siemens-Acuson Sequoia 512 scanner with a linear transducer 15L8w (Siemens-Acuson, Mountain View, CA) using contrast pulse sequencing $\left(\mathrm{CPS}^{\circledR}\right)$ technique. Before the examination, the subjects had to rest on in supine position in a darkened room for approximately 10 minutes. The triceps surae muscle of the primarily affected lower limb was examined. The transducer was placed over the gastrocnemius muscle of the subjects at the same supine position during the entire examination (Figure 1). Further details concerning the CEUS examination have been published by the authors $[1,2]$

The parameters were as follows: Transmit frequency, $7 \mathrm{MHz}$; MI, 0.28; gain, $75 \mathrm{~dB}$ and minimal persistence; depth, $40 \mathrm{~mm}$. Two foci were set at 2.25 and $3.5 \mathrm{~cm}$. A $4.8-\mathrm{mL}$ suspension with microbubbles containing sulphur hexafluoride (SonoVue ${ }^{\circledR}$, Bracco Imaging SpA., Milan, Italy) was continuously administered intravenously (infusion rate, $1 \mathrm{~mL} / \mathrm{min}$ ) within 5 min using a dedicated infusion pump (VueJect ${ }^{\mathbb{B}}$ BR INF-100, Bracco Imaging Germany, Konstanz, Germany). A 1.75 $\mathrm{cm} \times 5 \mathrm{~cm}$ sized region of interest (ROI) was positioned on recorded video clips (length, $5 \mathrm{~min}$; frame rate, $1 / \mathrm{s}$ ) at a depth of $2 \mathrm{~cm}$ within the gastrocnemius muscle. An automated occlusion plethysmograph Periquant ${ }^{\mathbb{B}} 803$ (Gutmann, Eurasburg, Germany) was used to simulate physical exercise: For this, a cuff was placed around the middle of the thigh and inflated with $50 \mathrm{mmHg}$ above arterial systolic pressure to induce transient arterial occlusion. This transient arterial occlusion is supposed to cause a hypoxic state within the muscle tissue that simulates the hypoxia experienced by PAOD patients during walking. The cuff was inflated $120 \mathrm{~s}$ after beginning of contrast media injection for 60 $s$ and then deflated $[1,2]$. The US-signal-intensity values in the ROI were calculated via the Axius ${ }^{\circledR}$ software installed in the scanner. The linearized data were exported as Excel file to a personal computer. A radiologist and physicist with 15 years of experience in musculoskeletal radiology and CEUS (M.K.) analyzed the TIC. Both were completely blinded to clinical information. In Figure 2 shows an example of a TIC: The maximum CEUS signal following the end of transient arterial occlusion $(\max )$, the time difference between the end of the occlusion and $\max \left(\mathrm{t}_{\max }\right)$ was calculated. Moreover, the maximum slope immediately after the occlusion $\left(\mathrm{m}_{2}\right.$, slope to $\left.\max \right)$ and the area under the curve after the occlusion $\left(\mathrm{AUC}_{\text {post }}\right)$ were evaluated [21-25].

\section{Statistical analysis}

Statistical analyses were performed using SPSS software (SPSS version 14.0, Chicago, Ill). CEUS parameters were evaluated by comparing mean values, standard deviations, and ranges. Student's t tests were used to evaluate significance. $\mathrm{P}$ value $<0.05$ was considered statistically significant. Pearson product-moment coefficients were 
Citation: Kauczor HU, Amarteifio E, Partovi S, Weber MA, Macys L, et al. (2016) Dynamic Quantitative Contrast-enhanced Ultrasound for Assessment of Cilostazol Induced Pro-angiogenic Therapeutic Effects on Skeletal Muscle Microcirculation in Peripheral Arterial Disease. J Vasc Med Surg 4: 284. doi: 10.4172/2329-6925.1000284

Page 3 of 6

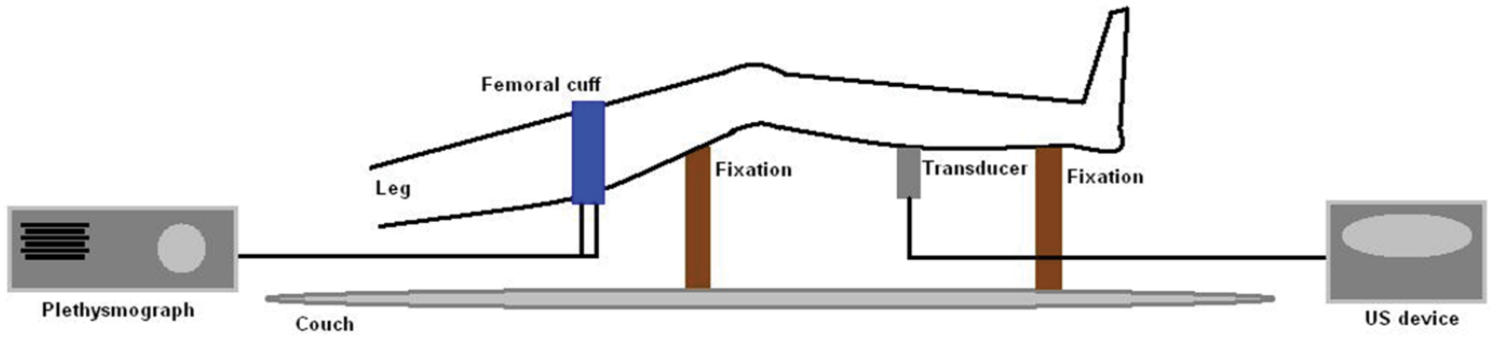

Figure 1: Illustration of the examination setting: The transducer was kept in the same position on the gastrocnemius muscle. With a transiently inflated cuff placed around the thigh and inflated approximately $50 \mathrm{mmHg}$ above the systolic blood pressure the arterial blood inflow was occluded.

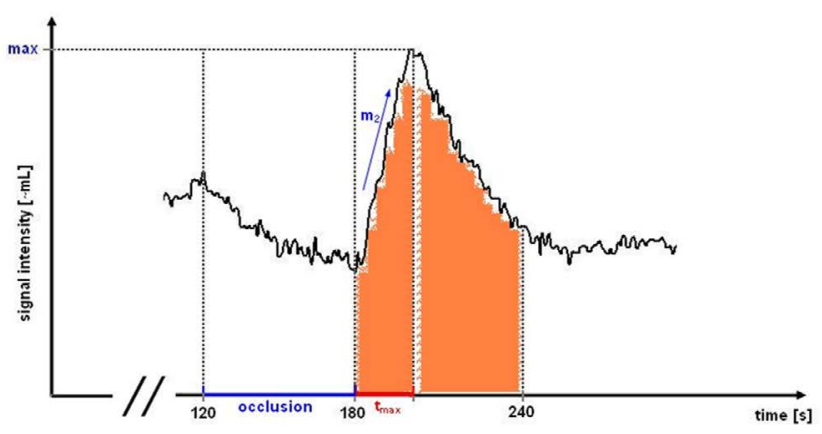

Figure 2: Signal-intensity-over-time curve. The following quantitative CEUS parameters were extracted from the curve: maximum signal during reperfusion $(\max )$, time to maximum signal after occlusion $\left(t_{\max }\right)$, maximum slope of reperfusion to $\max \left(\mathrm{m}^{2}\right)$, area under the curve post occlusion (AUCpost).

calculated to assess correlation of subjective clinical improvement and CEUS data prior toand after treatment. Coefficients $>0.5$ or $<-0.5$ indicate a moderately positive or negative correlation, respectively.

\section{Results}

\section{Clinical Endpoints}

The mean $\mathrm{ABI}$ in the dominant leg of the patients was $0.55 \pm$ 0.20 before and $0.62 \pm 0.20$ after therapy $(\mathrm{P}=0.09)$. The mean walking distance S2 prior and after cilostazol therapy for 3 months was $199.4 \pm$ 125.7 and $208.8 \pm 147.3 \mathrm{~m}(\mathrm{P}=0.85)$ and the mean walking distance $\mathrm{S} 1$ was $86.0 \pm 38.8 \mathrm{~m}$ and $163.9 \pm 157.6 \mathrm{~m}(\mathrm{P}=0.09)$, respectively. Details are summarized in Table 2.

\section{CEUS Parameters}

Maximum signal enhancement prior to treatment was $104 \pm 69$ $\sim \mathrm{mL}$ compared to $114 \pm 68 \sim \mathrm{mL}$ post treatment $(\mathrm{P}=0.26)$. Mean $\mathrm{t}_{\max }$ was $34 \pm 20 \mathrm{~s}$ before treatment, and demonstrating an increase post treatment $(36 \pm 23 \mathrm{~s})(\mathrm{P}=0.51)$. The slope to $\max \left(\mathrm{m}_{2}\right)$ prior to therapy was $6.06 \pm 5.55 \sim \mathrm{mL} / \mathrm{s}$ and $5.12 \pm 3.30 \sim \mathrm{mL} / \mathrm{s}$ post treatment $(\mathrm{P}=0.62)$. AUC $_{\text {post }}$ was $3975.38 \pm 2856.52 \sim \mathrm{mL} / \mathrm{s}$ prior compared to $4210.98 \pm$ $2816.33 \sim \mathrm{mL} / \mathrm{s}$ after treatment $(\mathrm{P}=0.39)$. The CEUS parameters for each patient are shown in Figure 3.

\section{Discussion}

To our knowledge this is the first published study analyzing the effect of cilostazol (Pletal ${ }^{\circledR}$ ) on muscular microperfusion in patients with PAOD with quantitative CEUS. After 3 months of treatment with cilostazol there was no significant difference between quantitative

\begin{tabular}{|c|c|c|c|c|}
\hline $\begin{array}{l}\text { Before } \\
\text { cilostazol }\end{array}$ & $A B I$ right leg & ABI left leg & $\begin{array}{l}\text { Walking dist. } \\
\text { S1 }\end{array}$ & $\begin{array}{l}\text { Walking dist. } \\
\text { S2 }\end{array}$ \\
\hline$\# 1$ & 0.31 (dominant) & 0.81 & 98 & 316 \\
\hline \#2 & 0.71 (dominant) & 0.42 & 75 & 117 \\
\hline \#3 & 0.7 (dominant) & 0.7 & 180 & 261 \\
\hline$\# 4$ & 0.88 & 0.66(dominant) & 77 & 469 \\
\hline$\# 5$ & 0.93 & 0.37 (dominant) & 70 & 113 \\
\hline \#6 & 0.9 & 0.3 (dominant) & 110 & 122 \\
\hline$\# 7$ & 0.71 (dominant) & 0.86 & 40 & 271 \\
\hline \#8 & 0.72 (dominant) & 1.2 & 75 & 96 \\
\hline$\# 9$ & 0.3 (dominant) & 0.5 & 85 & 144 \\
\hline$\# 10$ & 0.75 (dominant) & 1.2 & 50 & 85 \\
\hline mean & 0.69 & 0.7 & 86 & 199.4 \\
\hline After cilostazol & $\mathrm{ABI}$ right & $A B \mid$ left & Walking dist. S1 & Walking dist. S2 \\
\hline$\# 1$ & 0.46 (dominant) & 0.78 & 102 & 202 \\
\hline \#2 & 0.75 (dominant) & 0.4 & 293 & 191 \\
\hline$\# 3$ & 0.65 (dominant) & 0.84 & 570 & 600 \\
\hline$\# 4$ & 1 & 0.70 (dominant) & 105 & 215 \\
\hline$\# 5$ & 0.71 & 0.41 (dominant) & 93 & 126 \\
\hline$\# 6$ & 0.81 & 0.40 (dominant) & 72 & 100 \\
\hline$\# 7$ & 0.9 (dominant) & 1 & 155 & 250 \\
\hline$\# 8$ & 0.75 (dominant) & 1.2 & 77 & 113 \\
\hline$\# 9$ & 0.35 (dominant) & 0.47 & 112 & 191 \\
\hline$\# 10$ & 0.8 (dominant) & 1.2 & 60 & 100 \\
\hline Mean & 0.72 & 0.74 & 163.9 & 208.8 \\
\hline
\end{tabular}

Table 2: Clinical parameters before and after medication with cilostazol.

CEUS parameters appreciated indicating treatment response. The study demonstrated also the potential of CEUS to assess the potential therapeutic effect of Cilostazol on the microcirculatory level in the PAOD population [26-30].

Early studies confirmed the improvement of walking distance objectively as well as subjectively in patients with intermittent claudication by the use of cilostazol but none of these studies focused on the therapeutic effect of the medication on the microcirculatory level $[3,6]$. In our study, CEUS combined with the paradigm of transient arterial occlusion was used to evaluate skeletal muscle microperfusion in PAOD patients before and after treatment with cilostazol (3 months post starting of the medication). Walking distance test on a treadmill (constant workload treadmill test; $3.5 \mathrm{~km} / \mathrm{h} ; 12.5 \%$ ascending slope; S2 - the maximum walking distance in meters; S1 - free of pain walking distance in meters) showed a minimal, non-significant improvement whereas the quantitative CEUS parameters max and $\mathrm{AUC}_{\text {post }}$, reflecting the microperfusion of muscular tissue $[1,2]$ revealed no significant differences. The improvement of walking distance may have been secondary to modified nociception and treatment biased caused by 
Citation: Kauczor HU, Amarteifio E, Partovi S, Weber MA, Macys L, et al. (2016) Dynamic Quantitative Contrast-enhanced Ultrasound for Assessment of Cilostazol Induced Pro-angiogenic Therapeutic Effects on Skeletal Muscle Microcirculation in Peripheral Arterial Disease. J Vasc Med Surg 4: 284. doi: 10.4172/2329-6925.1000284

Page 4 of 6
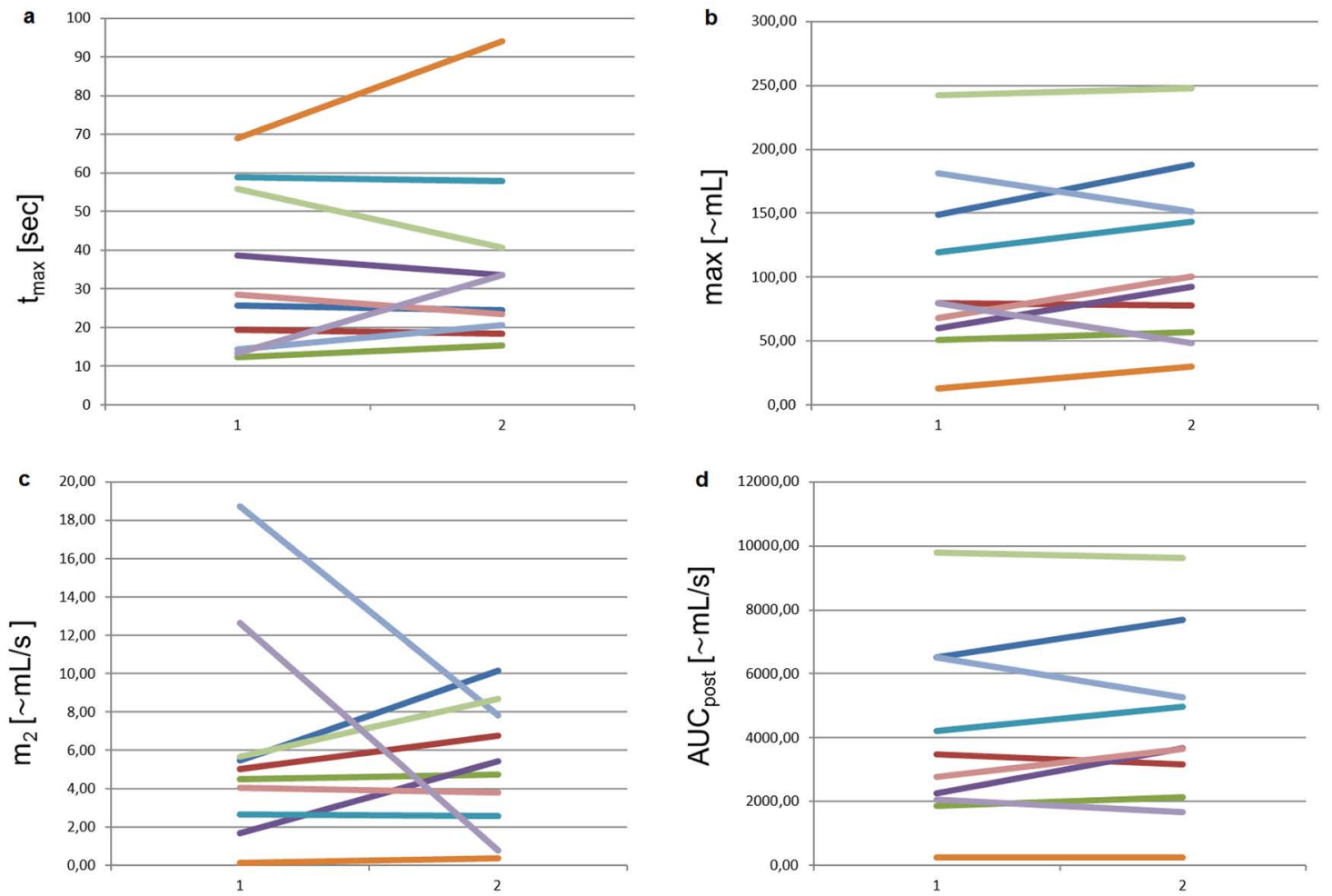

Figure 3: Contrast-enhanced ultrasound parameters of the subjects before and 3 months after medical treatment with cilostazol. a) tmax (time to maximum signal after occlusion), b) max (maximum signal during reperfusion), c) m2 (maximum slope of reperfusion to max), d) AUCpost (area under the curve post occlusion).

cilostazol. A previously published study is supporting this hypothesis and is demonstrating an effect of phosphodiesterase-III-inhibitors on nociception [1]. The authors of this study investigated the peripheral participation of the phosphodiesterase-III on formalin-evoked nociception and confirmed that peripheral phosphodiesterase-III influences (inflammatory) pain perception because it controls cyclic AMP levels and nociceptor threshold. Mechanisms of cilostazol effects on symptoms of intermittent claudication have not yet been completely elucidated [1] and therefore a potential effect on pain perception in PAOD for the subtle improvement of claudication symptoms based on the treadmill test results observed in our study has to be taken into consideration [31-35].

The parameters $\mathrm{m}_{2}$ and $\mathrm{t}_{\max }$ did not change significantly during the treatment with cilostazol. This was expected since they are primarily reflecting macroperfusion alterations which are not supposed to be influenced by cilostazol. Previous studies have demonstrated that the therapeutic effect of angioplasty, thrombendarterectomy and bypass surgery is assessable with these CEUS parameters $[2,3]$.

Decreased muscular capillary microperfusion has been established to be a hallmark of PAOD in a variety of studies. CEUS has been recently established as an evolving method to evaluate skeletal muscle microperfusion, particularly in the PAOD population [5-8].

CEUS has been proven to be a highly sensitive imaging technique for evaluation of muscular micoperfusion in humans. For example, muscle perfusion after transplantation of muscle flaps in plastic surgery can be monitored precisely [9]. Krix et al. showed that even minimal changes in capillary muscle micoperfusion can be appreciated applying
CEUS. The authors confirmed that CEUS can be used to show changes of muscular microperfusion determining quantitative levels in healthy volunteers during muscular work in real-time [5]. Based on these prior studies CEUS should be able to show treatment effects of cilostazol on the skeletal microperfusion level. Having said that, we did in our study only a three months follow-up and potentially longer follow-up times may be required to demonstrate a therapeutic effect of CEUS on the skeletal muscle microcirculation of PAOD patients. 10 out of the 20 enrolled patients exhibited significant side effects and had to be taken off the medication due to safety precautions. This is in accordance with the literature reporting marked risks associated with cilostazol medication, such as interaction with other medications, haemorrhage or dose-dependent cardiac arrhythmias [5]. One of the larger cilostazol studies, the CASTLE (Cilostazol: A Study in Long-term Effects) study was stopped at an early stage due to relevant numbers of dropouts treated with cilostazol and placebo [5].

\section{Limitations}

The main limitation of our study is its small sample size due to high drop out rate secondary to side effects of cilostazol. Stopping the medication in patients exhibiting side effects is in accordance with safety precautions and ethical standard. A second limitation was the short follow-up time of 3 months and it cannot be excluded that cilostazol improves skeletal muscle microcirculation after lincreased follow-up times. We decided to perform the CEUS evaluation 3 months post treatment based on the European medicine agency (EMA) recommendation to evaluate for therapeutic success after three months of medication with cilostazol. 
Citation: Kauczor HU, Amarteifio E, Partovi S, Weber MA, Macys L, et al. (2016) Dynamic Quantitative Contrast-enhanced Ultrasound for Assessment of Cilostazol Induced Pro-angiogenic Therapeutic Effects on Skeletal Muscle Microcirculation in Peripheral Arterial Disease. J Vasc Med Surg 4: 284. doi: 10.4172/2329-6925.1000284

The third limitation is the lack of a control group to assess the potential of CEUS to differentiate between PAOD patients receiving standard therapy versus PAOD patients receiving standars therapy plus Cilostazol. This is beyond the scope of this study and may be the topic of future investigations.

In conclusion, in this initial study CEUS with transient arterial occlusion as paradigm did not appreciate improvement of muscular microperfusion after therapy with cilostazol based on quantitative CEUS parameters. However the study showed the potential of CEUS to evaluate response to pro-angiogenic drug treatment. Confirmation of our results in a larger cohort study is warranted to further investigate the value of quantitative CEUS for assessment of the effect of cilostazol on the skeletal muscle microcirculation.

\section{Conflict of Interest}

This prospective study was investigator initiated. M. Krix is an employee of Bracco Imaging Germany, Konstanz, Germany. Otherwise the authors declare that they have no conflict of interest.

\section{References}

1. Chan D, Anderson ME, Dolmatch BL (2010) Imaging evaluation of lower extremity infrainguinal disease: role of the noninvasive vascular laboratory, computed tomography angiography, and magnetic resonance angiography. Techniques in vascular and interventional radiology 13: 11-22.

2. Tullos BW, Sung JH, Lee JE, Criqui MH, Mitchell ME, et al. (2013) Anklebrachial index $(\mathrm{ABI})$, abdominal aortic calcification (AAC), and coronary artery calcification (CAC): the Jackson heart study. The international journal of cardiovascular imaging 29: 891-897.

3. Wagner M, Knobloch G, Gielen M, Lauff MT, Romano V, et al. (2015) Nonenhanced peripheral MR-angiography (MRA) at 3 Tesla: evaluation of quiescent-interval single-shot MRA in patients undergoing digital subtraction angiography. The international journal of cardiovascular imaging 31: 841-850.

4. Aschwanden M, Partovi S, Jacobi B, Fergus N, Schulte AC, et al. (2014) Assessing the end-organ in peripheral arterial occlusive disease-from contrast-enhanced ultrasound to blood-oxygen-level-dependent MR imaging. Cardiovascular diagnosis and therapy 4: 165-172.

5. Wang JC, Criqui MH, Denenberg JO, McDermott MM, Golomb BA (2005) Exertional leg pain in patients with and without peripheral arterial disease. Circulation 112: 3501-3508.

6. Robless P, Mikhailidis DP, Stansby GP (2008) Cilostazol for peripheral arterial disease. Cochrane Database Syst Rev 24

7. Duerschmied D, Olson L, Olschewski M, Rossknecht A, Freund G, et al. (2006) Contrast ultrasound perfusion imaging of lower extremities in peripheral arterial disease: a novel diagnostic method. Eur Heart J 27: 310-315.

8. Lindner JR, Womack L, Barrett EJ, Weltman J, Price W, et al. (2008) Limb stress-rest perfusion imaging with contrast ultrasound for the assessment of peripheral arterial disease severity. JACC Cardiovasc Imaging1: 343-350.

9. Krix M, Weber MA, Kauczor HU, Delorme S, Roosen H (2010) Changes in the micro-circulation of skeletal muscle due to varied isometric exercise assessed by contrast-enhanced ultrasound. Eur J Radiol 76: 110-116.

10. Krix M, Krakowski-Roosen H, Kauczor HU, Delorme S, Weber MA (2009) Realtime contrast-enhanced ultrasound for the assessment of perfusion dynamics in skeletal muscle. Ultrasound in medicine \& biology 35: 1587-1595.

11. Krix M, Krakowski-Roosen H, Amarteifio E, Furstenberger S, Delorme S, et al. (2011) Comparison of transient arterial occlusion and muscle exercise provocation for assessment of perfusion reserve in skeletal muscle with realtime contrast-enhanced ultrasound. Eur J Radiol 78: 419-424.

12. Weber MA, Krix M, Jappe U, Huttner HB, Hartmann M, et al. (2006) Pathologic skeletal muscle perfusion in patients with myositis: detection with quantitative contrast-enhanced US initial results. Radiology 238: 640-649.

13. Otero HJ, Rybicki FJ, Greenberg D, Mitsouras D, Mendoza JA, et al. (2010) Cost-effective diagnostic cardiovascular imaging: when does it provide good value for the money? .The international journal of cardiovascular imaging 26 : $605-612$
14. Zhao H, Quilley J, Montrose DC, Rajagopalan S, Guan Q, et al. (2007) Differential effects of phosphodiesterase PDE-3/PDE-4-specific inhibitors on vasoconstriction and CAMP-dependent vasorelaxation following balloon angioplasty. Am J Physiol Heart Circ Physiol 292: H2973-2981.

15. Norgren L, Hiatt WR, Dormandy JA, Nehler MR, Harris KA, et al. (2007) InterSociety Consensus for the Management of Peripheral Arterial Disease (TASC II). J Vasc Surg 45: S5-67.

16. Takeda M, Yamashita T, Shinohara M, Sasaki N, Tawa H, et al. (2012) Beneficial effect of anti-platelet therapies on atherosclerotic lesion formation assessed by phase-contrast X-ray CT imaging. The international journal of cardiovascular imaging 28: 1181-91.

17. Hirsch AT, Haskal ZJ, Hertzer NR, Bakal CW, Creager MA, et al. (2006) ACC/ AHA 2005 Practice Guidelines for the management of patients with peripheral arterial disease (lower extremity, renal, mesenteric, and abdominal aortic) a collaborative report from the American Association for Vascular Surgery/ Society for Vascular Surgery, Society for Cardiovascular Angiography and Interventions, Society for Vascular Medicine and Biology, Society of Interventional Radiology, and the ACC/AHA Task Force on Practice Guidelines (Writing Committee to Develop Guidelines for the Management of Patients With Peripheral Arterial Disease): endorsed by the American Association of Cardiovascular and Pulmonary Rehabilitation; National Heart, Lung, and Blood Institute; Society for Vascular Nursing; TransAtlantic Inter-Society Consensus; and Vascular Disease Foundation. Circulation 113: e463-654.

18. Herman GM, Gardin JM, Jaff M, Mohler E, Roman M, et al. (2006) Guidelines for noninvasive vascular laboratory testing: a report from the American Society of Echocardiography and the Society for Vascular Medicine and Biology. Vasc Med 11: 183-200.

19. Emanuel EJ (2013) Reconsidering the Declaration of Helsinki. Lancet 381 : 1532-1533.

20. Rooke TW, Hirsch AT, Misra S, Sidawy AN, Beckman JA, et al. (2013) Management of patients with peripheral artery disease (compilation of 2005 and 2011 ACCF/AHA Guideline Recommendations): a report of the American College of Cardiology Foundation/American Heart Association Task Force on Practice Guidelines. J Am Coll Cardiol 61: 1555-1570.

21. Amarteifio E, Weber MA, Wormsbecher S, Demirel S, Krakowski-Roosen $\mathrm{H}$, et al. (2011) Dynamic contrast-enhanced ultrasound for assessment of skeleta muscle microcirculation in peripheral arterial disease. Invest Radiol 46: 504508

22. Amarteifio E, Wormsbecher S, Krix M, Demirel S, Braun S, et al. (2012) Dynamic contrast-enhanced ultrasound and transient arterial occlusion for quantification of arterial perfusion reserve in peripheral arterial disease. Eur $\mathrm{J}$ Radiol 81: 3332-3338.

23. Money SR, Herd JA, Isaacsohn JL, Davidson M, Cutler B, et al. (1998) Effect of cilostazol on walking distances in patients with intermittent claudication caused by peripheral vascular disease. J Vasc Surg 27: 267-274

24. Dawson DL, Cutler BS, Meissner MH, Strandness DE (1998) Cilostazol has beneficial effects in treatment of intermittent claudication: results from a multicenter, randomized, prospective, double-blind trial. Circulation 98: 678-86.

25. Beebe HG, Dawson DL, Cutler BS, Herd JA, Strandness DE, et al. (1999) A new pharmacological treatment for intermittent claudication: results of a randomized, multicenter trial. Arch Intern Med. 159: 2041-2050

26. Strandness DE, Dalman RL, Panian S, Rendell MS, Comp PC, et al. (2002) Effect of cilostazol in patients with intermittent claudication: a randomized double-blind, placebo-controlled study. Vasc Endovascular Surg 36: 83-91.

27. Amarteifio E, Krix M, Wormsbecher S, Demirel S, Braun S, et al. (2013) Dynamic contrast-enhanced ultrasound for assessment of therapy effects on skeletal muscle microcirculation in peripheral arterial disease: pilot study. Eur J Radiol 82: 640-646

28. Torres-Lopez JE, Granados-Soto V (2005) Peripheral participation of the phosphodiesterase 3 on formalin-evoked nociception. European journal of pharmacology 519: 75-79.

29. Pearce L, Ghosh J, Counsell A, Inglott SF (2008) Cilostazol and peripheral arterial disease. Expert opinion on pharmacotherapy 9: 2683-2690.

30. Duerschmied D, Maletzki P, Freund G, Olschewski M, Bode C, et al. (2010) Success of arterial revascularization determined by contrast ultrasound muscle perfusion imaging. J Vasc Surg 52: 1531-6. 
Citation: Kauczor HU, Amarteifio E, Partovi S, Weber MA, Macys L, et al. (2016) Dynamic Quantitative Contrast-enhanced Ultrasound for Assessment of Cilostazol Induced Pro-angiogenic Therapeutic Effects on Skeletal Muscle Microcirculation in Peripheral Arterial Disease. J Vasc Med Surg 4: 284. doi: 10.4172/2329-6925.1000284

Page 6 of 6

31. Partovi S, Kaspar M, Aschwanden M, Robbin MR, Bilecen D, et al. (2015) Quantitative dynamic contrast-enhanced ultrasound for the functional evaluation of the skeletal muscle microcirculation in systemic sclerosis. Clinical hemorheology and microcirculation.

32. Schinkel AF, Kaspar M, Staub D (2015) Contrast-enhanced ultrasound: clinical applications in patients with atherosclerosis. The international journal of cardiovascular imaging.

33. Lamby P, Prantl L, Fellner C, Geis S, Jung EM (2011) Post-operative monitoring of tissue transfers: advantages using contrast enhanced ultrasound (CEUS) and contrast enhanced MRI (ceMRI) with dynamic perfusion analysis? Clinical hemorheology and microcirculation 48: 105-117.

34. NCBI (2009) Cilostazol: new drug. Intermittent claudication: too little efficacy, too many risks. Prescrire international 18: $56-59$

35. Hiatt WR, Money SR, Brass EP (2008) Long-term safety of cilostazol in patients with peripheral artery disease: the CASTLE study (Cilostazol: A Study in Longterm Effects). JVasc Surg 47: 330-336. 